\title{
Author Correction: Inefficient splicing curbs noncoding RNA transcription
}

Noa Gil(D) and Igor Ulitsky (D)

Correction to: Nature Structural \& Molecular Biology https://doi.org/10.1038/s41594-021-00582-w, published online 25 March 2021.

In the version of this article initially published online, the authors' affiliations were swapped. The correction affiliation for Noa Gil is Friedrich Miescher Institute for Biomedical Research, Basel, Switzerland, and the correct affiliation for Igor Ulitsky is Department of Biological Regulation, Weizmann Institute of Science, Rehovot, Israel. The error has been corrected in the print, PDF and HTML versions of the article.

Published online: 1 April 2021

https://doi.org/10.1038/s41594-021-00588-4

(C) The Author(s), under exclusive licence to Springer Nature America, Inc. 2021 